FACTA UNIVERSITATIS

Series: Mechanical Engineering Vol. 17, N N $^{\mathrm{o}}$, 2019, pp. 39 - 45

https://doi.org/10.22190/FUME190112007P

Original scientific paper

\title{
GENERALIZED ARCHARD LAW OF WEAR BASED ON RABINOWICZ CRITERION OF WEAR PARTICLE FORMATION
}

\begin{abstract}
Valentin Popov
Technische Universität Berlin, Berlin, Germany

Institute of Strength Physics and Materials Science, Russian Academy of Sciences

Abstract. According to the Archard law of adhesive wear, the wear volume is proportional to the normal force, the sliding distance, and inversely proportional to the hardness of the softer of contact partners. This law does not contain any properties characterizing "adhesion" of materials, e.g. the work of separation, either inside of the material or at the interface. The criterion for formation of wear particles, first formulated by Rabinowicz in 1958, on the contrary, is based on the interplay of elastic energy and work of adhesion and contains as governing parameters the modulus of elasticity, hardness and the work of separation. Following recent advances in understanding and simulation of wear, we discuss the ways how both laws could be melted together to a "generalized" Archard-Rabinowicz law of wear.
\end{abstract}

Key words: Adhesive wear, Archard, Rabinowicz, Particle formation, Roughness

\section{INTRODUCTION}

In 1953, Archard published an article with the modest title "Contact and Rubbing of Flat Surfaces" [1], in which he analyzed the contact of rough surfaces and on this basis, among other things, formulated the law of adhesive wear. This law is very simple and states that the volume $V$ of worn material is proportional to the normal force, $F$, the sliding distance, $s$, and inversely proportional to the hardness of the material, $\sigma_{0}$ :

$$
V=k_{\text {adh }} \frac{F s}{\sigma_{0}},
$$

The constant $k_{\text {adh }}$ is the so-called adhesive wear coefficient.

Received January 12, 2018 / Accepted March 01, 2018

Corresponding author: Valentin L. Popov

Technische Universität Berlin, Sekr. C8-4, Straße des 17. Juni 135, 10623 Berlin, Germany

E-mail: v.popov@tu-berlin.de 
As the law of Amonton-Coulomb for the force of friction, this is of course only a very rough empirical estimation. It is used primarily because of its simplicity and due to the absence of a generally accepted alternative. There exists a widespread opinion that the higher the hardness, the smaller is the wear, since the hardness is in the denominator of the Archard law. This would be true if it were not for the coefficient of adhesive wear, which stands in the Archard law as a multiplier. In reality, this coefficient can take values that differ by at least seven orders of magnitude, which deprives the law of any predictive power. Indeed, Kragelsky [2] formulated the exact opposite condition of low wear - the principle of a positive gradient of hardness, which states that the surface layers should be softer than the deeper ones, otherwise catastrophic wear will occur.

In the problem of wear, not only the total worn volume is of interest, but also the distribution of wear particles by size. Wear debris formation and transportation is, after all, the physical mechanism of all types of "mesoscopic" wear (contrary to chemical, atom-by-atom wear). Archard's law says nothing about the particle size. The first researcher who investigated this topic was Ernest Rabinowicz. In 1958, he wrote a five page-article in Wear, in which he put forward the hypothesis of a mechanism determining the size of wear particles [3, 4]. He considered two micro-heterogeneities which collide and form a bridge, as suggested by Bowden and Tabor [5]. Due to the plastic properties of the material, the maximum stress that can be achieved in this case is on the order of magnitude of the material hardness, $\sigma_{0}$. Elastic energy stored in the material is proportional to the third degree of the size $D$ of the contact: $\left(\sigma_{0}^{2} / 2 G\right) D^{3}$, where $G$ is shear modulus of the medium. This energy can relax by dislodging wear particles, but only if the stored elastic energy is larger than the energy needed to create new free surfaces, $w D^{2}$, where $w$ is the specific work of separation:

$$
\frac{\sigma_{0}^{2}}{2 G} D^{3} \geq w D^{2} .
$$

From this energetic criterion, it follows that only particles with a size larger than a certain critical size can detach spontaneously:

$$
D \geq D_{c}=2 G \frac{w}{\sigma_{0}^{2}} .
$$

The details of how plastic deformation and detachment occur during the wear process were not clear at that time. Only 58 years later, in 2016, Aghababaei, Warner and Molinari published an article in Nature Communications [6], which returns to the idea of Rabinowicz, but at the level of physical mechanisms. Aghababaei and colleagues did a very simple thing: they implemented Rabinowicz' thought experiment in a mesomolecular model by generating two bodies with micro asperities. They forced the asperities to collide and carefully observed the results of their simulations. The medium generated by Aghababaei and co-authors did not correspond to any real material, but it contained all the parameters that enter into the Rabinowicz' equation: elastic modulus, specific work of separation and hardness. The behavior of asperities at impact did depend on the size of asperities. If the initial size of asperities was small enough, the main process was plastic deformation and gradual smoothing of the roughness. The process proceeded in a completely different way when two large asperities collided. In this case, from the very 
beginning, the process of cracking and formation of wear particles dominated. The breakthrough work by Aghababaei, Warner and Molinari created a solid concept basis for model-based simulation of wear. The work of Aghababaei, Warner and Molinari confirmed the Rabinowicz criterion and lead to appearance of a new paradigm as represented by the recent works [7-9].

In particular, in [8], a generalization of the Rabinowicz' criterion to heterogeneous media (e.g. layered materials) and in [9] an "asperity free" generalization of the Rabinowicz' criterion have been suggested. This latter generalization is a principal step forward as the asperity size is a very poorly defined quantity for multiscale surface topographies. The approach suggested in [9] allowed the simulation of the development of the surface topography during the wear process. The simulation procedure included numerical determination of the contact regions having enough elastic energy for dislodging a particle of corresponding size with its subsequent "disappearance" from the system. The detachment of a particle changes the topography so that the critical conditions are created at another position. This process leads to an overall change in surface topography. After running the system for a long time, one can look at the distribution of particle sizes or calculate the total amount of wear. Simulations carried out in [9] revealed a number of regimes. Depending on the normal pressure and initial topography, the authors observed different regimes of wear from settling type to continuous and catastrophic wear. In the region of "mild wear", which is the main interest in the present study, a power-law dependency of the worn volume on the normal load was found which corresponds to experimental findings. Indeed, the non-linear dependencies of the worn volume on the normal force in the case of large force interval have been reported as early as in 1970s [10]. An extensive experimental analysis of polymer materials can be found in [11] and a review for very broad material classes in [12].

It is important to note that the deviations from the Archard law of wear (proportionality of wear volume to the normal force) may be the key to solving the riddle of the huge variation in the coefficient of adhesive wear. Indeed, is it not paradoxical that Archard's equation, which describes adhesive wear, does not contain any parameter characterizing adhesion? Moreover, from considerations of dimension, it follows that the coefficient of wear cannot depend on the specific work of adhesion, since the available parameters do not allow building a dimensionless combination. The situation would change qualitatively if wear would be nonlinear with force. Then the specific work of separation could be included in the equation of wear in a natural way.

In the present paper, we analyze the possible power-law dependencies on system parameters. We consider stationary wear and two main modes of wear particle transport corresponding to open and closed tribological systems for both elastic and elastoplastic media.

\section{POWER LAW WEAR EQUATIONS}

We distinguish between the initial stage of wear, when surfaces with given surface topography are brought into contact and then forced in relative tangential movement and the state of stationary wear after the running-in stage. Here, we focus on the stage of stationary wear. The current surface topography determines the contact configuration and 
the detailed stress distribution at any time moment and thus determines the position and the size of wear particles (e.g. according to rules sketched in [9]). However, the wear process changes the surface topography, so that the properties of the surface topography cannot be considered as given but are also a product of the tribological process. Following this line of argumentation, Rabinowicz [13] came to the conclusion that the stationary roughness of contacting surfaces is on the same order of magnitude as the critical size (3) of junctions. As a matter of fact, it is not just the "roughness" but the "surface topography" which is determined by the characteristic length, Eq. (3). In the paper [14] of the present author, it was argued that formation of the near-surface properties can be considered as a key problem and a great current challenge of tribology. At the present, no recognized concepts exist of how "friction machine" leads to formation of the stationary topography, or more generally, to formation of the third body. We only assume at this point that such stationary state does exist and that all its properties including rms roughness, wavelength cutoffs and others do not represent independent properties but are all functions of material properties.

Wear is not only the problem of cracking and detachment of wear debris but also of their transport out of the wear zone. In the present paper, we consider two cases:

1. The case of "immediate disappearance" of wear particles. This is the case considered in the majority of theories of wear. Physically, it corresponds to the case of an open system in which the wear region regularly is cleaned from debris or wear particles have opportunity to leave the frictional zone. In this case, it is reasonable to assume that the wear is occurring homogeneously in the whole contact area, so that the wear intensity depends on the pressure and the wear volume is proportional to the square $L^{2}$ of the size $L$ of the contact region.

2. The case of continuous transport of wear debris towards the boundary of the contact. This is typical condition for a closed tribological system. This diffusion like process can be accompanied be reintegration of the particles into the surface of by transfer of material from one partner to the other. Under assumption of the existence of some characteristic size of wear particles, it was shown in [15] that the wear intensity (defined as the volume of the worn material per unit sliding length) at the given force is inversely proportional to the square of the size of the contact region. Correspondingly, at a given pressure it does not depend on the size of the contact. (It is proportional to $L^{2}$ due to homogeneity of the process and to $L^{-2}$ due to "diffusion like" transport of wear debris, so that both factors cancel each other).

\subsection{Immediate disappearance of debris (elastoplastic materials)}

Our main intention is to modify the Archard wear equation in such a way that the critical Rabinowicz' length (3) comes into play. As explained above, it is necessary that the dependency on the normal force becomes non-linear; we thus assume a power-law dependency with some exponent $\alpha$. We search for a dependency in the form of a product of powers of all relevant governing parameters:

$$
V=k p^{\alpha} \sigma_{0}^{\beta} D_{c}^{\delta} L^{2} s
$$

where $p$ is the apparent pressure in the contact region, $\sigma_{0}$ is the hardness, $D_{c}$ the Rabinowicz' critical length, $L$ the linear size of the system and $s$ the sliding distance. 
The main assumptions behind this form are the following:

- We consider the stationary wear, therefore the wear volume is proportional to the sliding distance, $s$.

- The wear process occurs homogeneously in the whole contact area. Therefore, the wear intensity is proportional to $L^{2}$ and is determined locally by the pressure, $p=$ $F / L^{2}$.

- All geometrical parameters of the contacting surfaces and thus the stationary wear process are determined solely by the Rabinowicz' critical length, $D_{c}$.

From dimensional analysis, it follows that $\delta=0, \alpha=-\beta$ which leads to the following modified form of Archard's wear law (1):

$$
V=k\left(\frac{p}{\sigma_{0}}\right)^{\alpha} L^{2} s=k\left(\frac{F}{\sigma_{0}}\right)^{\alpha} L^{2(1-\alpha)} s .
$$

We see that the assumption of a stationary homogeneous wear intensity is compatible with a non-linear dependency of the wear intensity on the normal force, however, only at the expense of an additional dependency of the wear intensity on the system size. However, the dependence on adhesive properties of material is still absent.

\subsection{Continuous transport of debris (elastoplastic materials)}

According to the comments above, we search in this case for the wear law in the form

$$
V \propto p^{\alpha} \sigma_{0}^{\beta} D_{c}^{\delta} s .
$$

From the dimensional analysis, it follows: $\alpha+\beta=0, \delta=2$, or explicitly

$$
V=k\left(\frac{F}{L^{2} \sigma_{0}}\right)^{\alpha} G^{2} \frac{w^{2}}{\sigma_{0}^{4}} s=k F^{\alpha} L^{-2 \alpha} \sigma_{0}^{-4-\alpha} G^{2} w^{2} s .
$$

If $\alpha=1$, so that the classical Archard's law (proportionality to the normal force) is valid, it follows for the adhesive wear coefficient

$$
k_{\text {adh }}=k\left(\frac{G w}{L \sigma_{0}^{2}}\right)^{2} \propto\left(\frac{D_{c}}{L}\right)^{2} .
$$

According to this equation, the wear coefficient is inversely proportional to the size of the system (as suggested in [15]) and is determined by the square of the ratio of the Rabinowicz' critical length to the system size.

\subsection{Immediate disappearance of debris (elastic materials)}

As illustrated in paper [9], the application of the ideas behind the Rabinowicz' criterion is not restricted to materials which can be deformed plastically. If the material is purely elastic and the tangential stresses which appear in the contact are characterized by the coefficient of friction, then the conditions for detaching of wear particles can still be 
fulfilled. In this case however, the hardness is not available as a governing parameter. Similarly to the Section 2.1., we assume the following:

- We consider the stationary wear, therefore the wear volume is proportional to the sliding distance, $s$.

- The wear process occurs homogeneously in the whole contact area. Therefore, the wear intensity is proportional to $L^{2}$ and is determined locally by the pressure, $p=F / L^{2}$.

$$
V \propto p^{\alpha} G^{\beta} w^{\delta} L^{2} s .
$$

From the dimensional analysis, it follows: $\alpha+\beta=0, \delta=0$. Thus, the wear intensity again does not depend on the work of separation:

$$
V=k\left(\frac{F}{G}\right)^{\alpha} L^{2-2 \alpha} s .
$$

In the simplest case of linear proportionality to the normal force, we will get

$$
V=k \frac{F s}{G} .
$$

Let us also discuss what happens if we renounce the claim that the wear intensity is proportional to the area, $L^{2}$, but still assume stationary wear (and thus direct proportionality to $s$ ). The general form of the power-law dependency is under these assumptions

$$
V \propto F^{\alpha} G^{\beta} L^{\gamma} w^{\delta} s .
$$

Dimensional analysis gives:

$$
\delta=2-\gamma-2 \alpha, \quad \beta=\alpha+\gamma-2
$$

and

$$
V=k F^{\alpha} L^{\gamma} G^{\alpha+\gamma-2} w^{2-\gamma-2 \alpha} S
$$

which finally brings in play also the specific work of separation (compare with [11]).

\section{DISCUSSION}

In the present paper we analyzed power-law wear equations under conditions of stationary wear. We find that under the additional assumption of homogeneity of wear in the contact plane, the work of separation does not enter the wear equation. Only deviation from this bound (for example due to transport of wear particles) makes the dependency of wear intensity on the specific work of separation possible. With other words, the specific work of separation can only enter the wear equation provided the wear process is characterized by some characteristic length. This can be the characteristic Rabinowicz' length or other structural parameter. It is extremely interesting to check the found dependencies both experimentally and using direct numerical simulations as suggested in [9]. 
Acknowledgement: This work has been conducted under partial financial support from the German ministry for research and education BMBF, grant No. 13NKE011A and by the Russian Science Foundation (project 17-11-01232).

\section{REFERENCES}

1. Archard, J. F., 1953, Contact and Rubbing of Flat Surfaces, Journal of Applied Physics, 24, pp. 981-988.

2. Kragelski, I.V., 1965, Friction and Wear, Butter Worth.

3. Rabinowicz, E., 1958, The effect of size on the looseness of wear fragments, Wear, 2, pp. 4-8.

4. Popova, E., Popov, V.L., Kim, D.-E., 2018, 60 years of Rabinowicz' criterion for adhesive wear, Friction, 6(3), pp.341-348.

5. Bowden, F.P., Tabor, D., 2001, The Friction and Lubrication of Solids, Clarendon Press.

6. Aghababaei, R., Warner, D.H., Molinari, J.-F., 2016, Critical length scale controls adhesive wear mechanisms. Nature Communications. 7, 11816.Rabinowicz, E., 1995, Friction and wear of materials. Second Edition, John Wiley \& Sons, inc.,

7. Aghababaei, R., Warner, D.H., Molinari, J.-F., 2017, On the debris-level origins of adhesive wear, Proceedings of the National Academy of Sciences, 114(30), pp. 7935-7940.

8. Popov, V.L., 2018, Adhesive wear: Generalized Rabinowicz' criteria, Facta Universitatis-Series Mechanical Engineering, 16(1), pp. 29-39.

9. Popov, V.L., Pohrt, R., 2018, Adhesive wear and particle emission: Numerical approach based on asperity-free formulation of Rabinowicz criterion, Friction, 6(3), pp.260-273.

10. Rhee, S K., 1970, Wear equation for polymers sliding against metal surfaces, Wear, 16(6), pp. 431-45.

11. Kar, M K., Bahadur, S. 1974, The wear equation for unfilled and filled polyoxymethylene, Wear, 30(3), pp. 337-348.

12. Meng, H.C., Ludema, K.C., 1995, Wear models and predictive equations: their form and content, Wear, 181183, pp.443-457.

13. Rabinowicz, E., 1995, Friction and wear of materials. Second Edition, John Wiley \& Sons, inc.

14. Popov, V.L., 2018, Is Tribology Approaching Its Golden Age? Grand Challenges in Engineering Education and Tribological Research, Frontiers in Mechanical Engineering, 4, 16.

15. Popov, V.L., Smolin, I.Yu., Gervé, A., Kehrwald, B., 2000, Simulation of wear in combustion engines, Computational Materials Science, 19, pp. 285-291. 